\title{
A Seismic Signal Denoising Method Based on Wavelet Comprehensive Threshold
}

\author{
Jingsong Yang, Jie Lia, Han Wang \\ Institute of Disaster Prevention, Vibration Key Laboratory of Precision Measurement Technology and Instrument065201, China
}

\begin{abstract}
The wavelet comprehensive threshold is used to inherit and develop the advantages of hard threshold and soft threshold denoised method. Based on the small wavelet coefficients of the noise signal and the large wavelet coefficients of the seismic signal, the simulation experiment of the Ricker signal and the noise reduction experiment is carried out. The simulation results show that the MSE of the wavelet comprehensive threshold is the closest to the original signal waveform after noise reduction, and the energy of the high frequency part of the waveform is reduced and the low frequency part is suppressed. Finally, the actual seismic waveform, for example, the noise reduction of the actual waveform can be the first time of the waveform, and the waveform of the effective signal energy, noise signal energy is suppressed.
\end{abstract}

\section{Introduction}

In the process of seismic signal acquisition, it is affected by external environment and the uncertainty of the sensor itself. The signal is mixed with secondary noise, environmental noise and instrument noise, which leads to the occurrence of earthquake misjudgment and missed judgment (Zheng Zuoya et al., 2007; Fan Tao, 2014). Among the three types of noise, instrument noise has a weak influence on seismic signals, so it mainly reduces the misjudgment and missed judgment by eliminating the interference caused by secondary noise and environmental noise (Li Ying et al., 2006).

For the problem that wavelet threshold denoising has soft and hard threshold function which can not effectively eliminate the influence of noise signal on seismic signal, the wavelet integrated threshold method is proposed to improve the threshold function. After the improvement, the wavelet coefficients of the wavelet integrated threshold function are infinitely close to the wavelet coefficients of the real function, which not only maintains the continuity of the signal but also preserves the high frequency signal to achieve noise reduction.

\section{Wavelet integrated threshold denoising method}

\subsection{Wavelet integrated threshold denoising method principle}

Threshold function method is also called wavelet threshold denoising method. Donoho et al. have demonstrated that wavelet threshold denoising methods are superior to other classical noise reduction methods (Bruni et al., 2006). Currently, commonly used threshold noise reduction methods include soft and hard threshold denoising methods. The hard threshold method compares the absolute value of the signal wavelet coefficient with the threshold of the wavelet coefficient to achieve the preservation of the wavelet coefficients in the high-frequency part of the signal, but the discontinuity is likely to occur at the threshold zero, resulting in the absence of effective signals ( Chong et al, 2015; Mousavi et al.2016), the hard threshold function is shown in formula (1); the soft threshold method improves the signal missing phenomenon in the hard threshold method, but loses the high-frequency signal energy, and the soft threshold function is as shown in formula (2).

$$
\begin{gathered}
\operatorname{hard}(\omega, \lambda)=\left\{\begin{array}{l}
\omega(|\omega| \geq \lambda) \\
0(|\omega|<\lambda)
\end{array}\right. \\
\operatorname{soft}(\omega, \lambda)=\left\{\begin{array}{l}
\operatorname{sgn}(\omega)(|\omega|-\lambda)(|\omega| \geq \lambda) \\
0(|\omega|<\lambda)
\end{array}\right.
\end{gathered}
$$

In the formula $w$ is the signal wavelet coefficient; $\quad \lambda$ is the threshold value (non-negative value); $\operatorname{sgn}$ is the symbol function, when the noisy signal is greater than 0 , $\operatorname{sgn}=1$; when the noisy signal is less than 0 , $\operatorname{sgn}=-1$. Aiming at the insufficiency of high-frequency signal energy loss in the process of noise reduction by soft threshold method, wavelet comprehensive threshold method combined with hard threshold method to improve the high-frequency part of the signal and use the soft threshold method to maintain signal continuity in the low-frequency part of the signal, propose wavelet The integrated threshold function is shown in equation (3). 


$$
\operatorname{new}(\omega, \lambda)=\left\{\begin{array}{l}
\omega(|\omega| \geq \lambda) \\
\operatorname{sng}(\omega)(|\omega-\lambda|)(\omega \leq \lambda)
\end{array}\right.
$$

where $\omega_{j, k}$ is the true wavelet coefficient, $\hat{w}_{j, k}$ is the improved wavelet coefficient, To improve the wavelet coefficient, $\quad b=\frac{\left|w_{j, k}\right|-\lambda}{\left|w_{j, k}\right|}$ when $\left|w_{j, k}\right|=\lambda, \quad \mathrm{b}=0, \quad \hat{w}_{j, k}=0$, solve the hard threshold method in the threshold zeroing part of the discontinuity phenomenon. With the increase of $\left|w_{j, k}\right|, \mathrm{b}=1$ and $\hat{w}_{j, k} \approx w_{j, k}$. Solve the problem of high-speed signal energy loss in the soft threshold method. Wavelet integrated threshold denoising preserves the high frequency signal while changing the threshold function while maintaining signal continuity. Experimental results show that the improved threshold function has a good effect on seismic signal noise reduction.

SNR (Signal to Noise Ratio) and MSE (Average Variance) are a way to evaluate the merits of the noise reduction method. Assuming the seismic signal vector is $\mathrm{a}=\left[\mathrm{a}_{0}, \mathrm{a}_{1}, \mathrm{a}_{2}, \ldots \mathrm{a}_{\mathrm{N}-1}\right]^{\mathrm{T}}$, there is a formula (4):

$$
a_{i}=f_{i}+n_{i} \quad i=0,1,2, \ldots N-1
$$

where $f_{i}$ is the function $f$ Sampling, $n_{i}$ is a Gaussian white noise distributed $N(0, \sigma)$. The goal of noise reduction is that the mean variance MSE of the fitted value $\hat{f}$ is the smallest, and the expression of MSE is as shown in formula (5)

$$
R(\hat{f}, f)=\frac{1}{N}\|\hat{f}-f\|^{2}=\frac{1}{N} \sum_{i=0}^{N-1}\left(\hat{f}_{i}-f_{i}\right)^{2}
$$

From equation (5), the smaller the $\hat{f}_{i}-f_{i}$ is, the smaller the MSE value is, after noise reduction. The closer the waveform is to the original signal waveform.

\subsection{Wavelet characteristic energy spectrum coefficient}

Wavelet characteristic energy spectrum coefficient is a representation of the noise reduction method. It can visually observe the energy distribution of the signal in the low frequency and high frequency parts, and it is convenient to observe the noise reduction result and draw conclusions quickly. The wavelet characteristic energy spectrum coefficients are decomposed by i scales and the total energy is unchanged, as in formula (6), where $f(n)$ is the discrete sampling sequence of the seismic signal, A is the low frequency part of the signal, and D is the high frequency part of the signal, $\mathrm{A}_{\mathrm{i}} f(n), D_{i} f(n)$, is the component of each frequency after the scale transformation, $E_{i}^{A} f(n), E_{i}^{D} f(n)$ is the low-frequency signal component energy and the high-frequency signal component energy at the decomposition scale $i$, respectively.

$$
\begin{gathered}
f(n)=A_{i} f(n)+D_{i} f(n)+\ldots+D_{i} f(n) \\
E_{i}^{A} f(n)=\sum_{i=1}^{N}\left(A_{i} f(i)\right)^{2} \\
E_{i}^{D} f(n)=\sum_{i=1}^{N}\left(D_{i} f(n)\right)^{2}, i=1,2, \ldots, N
\end{gathered}
$$

Define the ratio between the energy of each component in the wavelet decomposition and the total energy, that is, the wavelet characteristic energy spectrum coefficient, expressed by the parameters $h E_{i}^{A}$ and $h E_{i}^{D}$, respectively:

$$
h E_{i}^{A}=\frac{E_{i}^{A} f(n)}{E f(n)}, h E_{i}^{D}=\frac{E_{i}^{D} f(n)}{E f(n)} j=1,2, \ldots, N
$$

\section{Wavelet integrated threshold noise reduction experiment}

\subsection{Simulated seismicsignal experiment}

denoising

The experimental research processed signal is for the down hole near-seismic signal frequency band. Then ear-shock signal is coif wavelet as the wavelet basis function, and the wavelet characteristic energy spectrum coefficient of the signal after 6 decompositions is calculated. Observed near the characteristic energy spectrum coefficient at the third decomposition scale the seismic signal energy is strong, so the wavelet characteristic energy spectrum coefficient on the third decomposition is selected. The down-seismic near-seismic signal acquisition process includes near-seismic signals and noise signals. The frequency of the peak energy of the near-seismic signals is mainly concentrated at 3-6 Hz. According to the random noise source and the performance law of the noise itself, the noise is divided into three categories (Table 1):

Table 1 Noise classification

\begin{tabular}{ccc}
\hline Noise type & $\begin{array}{c}\text { Frequency range } \\
(\mathrm{Hz})\end{array}$ & $\begin{array}{c}\text { Noise reduction } \\
\text { difficulty }\end{array}$ \\
\hline Environmental noise & $3-20$ & Difficult \\
Secondary noise & $5-30$ & Difficult \\
Instrument noise & $1-2$ & Easy \\
\hline
\end{tabular}

In order to compare the noise reduction ability of the wavelet integrated threshold method and the soft threshold method for secondary noise and environmental noise, select the near-shock signal. The Rake wavelet signal with similar wavelet coefficient characteristics was simulated. The Rake wavelet signal adds a noise frequency range of 3-30 Hz, including ambient noise and secondary noise. Fig.1(a) shows the waveform of the Rake wavelet and the waveform of the noise-reconciled wavelet. The soft threshold and wavelet integrated threshold denoising experiment of the noisy rake wavelet

a Corresponding author: 1009278271@qq.com 
waveform are shown in Fig. 1(b).

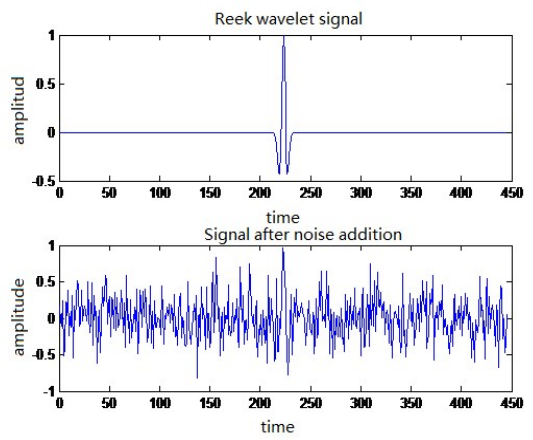

(a) The rexson waveform and the noised waveform
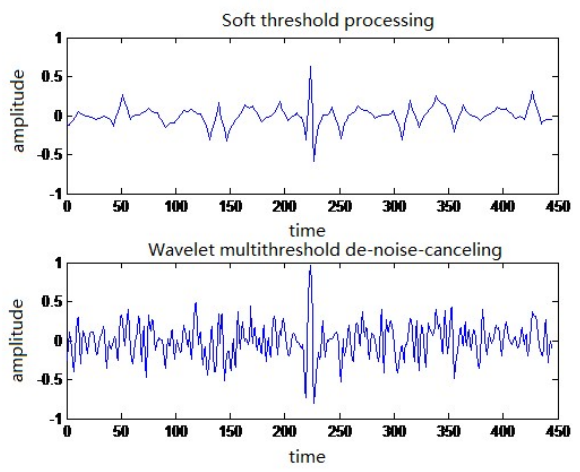

(b) Wave form comparison of the results of the two threshold noise reduction methods

Figure 1. Rexson waveform and noise reduction waveform

In Figure 1(a), the signal of the Rake wavelet after the noise is $210 \mathrm{~s}$ at the first arrival. After the noise is added, the high-frequency signal of the waveform is suppressed, and the seismic signal and the noise signal cannot be distinguished. Comparing the waveforms of the two methods of noise reduction in Figure 1(b), the soft threshold method suppresses the amplitude of the high-frequency signal. The noise near the zero-frequency is similar to the waveform after the noise-added, and the noise reduction effect is not obvious. The wavelet comprehensive threshold method improves the high-frequency signal. Amplitude reduces the amplitude of noise at zero frequency. By calculating the SNR and MSE (Table 2), the wavelet integrated threshold method is improved on two indicators. The wavelet integrated threshold method has the smallest MSE value after noise reduction, and the signal after noise reduction is more similar to the original signal.

Table 2 SNR and MSE from the de-noised signal in simulation

\begin{tabular}{ccc}
\hline The noise reduction method & SNR & MSE \\
\hline Hard threshold & 18.5736 & 0.2907 \\
Soft threshold & 19.3285 & 0.2897 \\
Wavelet synthesis threshold & 19.4136 & 0.2784 \\
\hline
\end{tabular}

\section{Results and discussion}

The wavelet integrated threshold denoising method uses the hard threshold method to improve the high-frequency signal energy, and uses the soft threshold method to preserve the signal smoothness of the low-frequency part of the signal, and improves the signal de-noising ability while ensuring signal continuity. The soft threshold, the wavelet integrated threshold method and the soft threshold method based on $\mathrm{S}$ transform are used to denoise the signal to observe the noise reduction. Experiments show that the wavelet high-frequency signal is recovered and the noise energy spectrum coefficient is suppressed by the wavelet integrated threshold method. The waveform MSE value is the smallest after the wavelet comprehensive threshold denoising, and the waveform after noise reduction is the closest to the original signal waveform. The noise reduction effect is as obvious as the soft threshold denoising method based on $\mathrm{S}$ transform. However, the wavelet comprehensive threshold method has a lot of problems of slow data processing, and the redundancy problem existing in the algorithm should be improved or the improvement of the threshold function should be further proposed. The improved wavelet integrated threshold method should be able to adapt to signals with similar characteristics to the near-seismic signals, increasing the breadth and universality of the application.

\section{Acknowledgments}

This work was funded by the department of Science and technology of Hebei province (Grant No. 16275417); China Scholarship Council.

\section{References}

1. Wei Xueqiang, Yuan Hongke, Qin Jingjing, Zuo Ying. Time-frequency analysis of generalized s-transform seismic signals [J]. Earthquake disaster prevention technology, 11 (2017): 808-813.

2. Geng Guanshi, Yu Yanxiang. Empirical relationship between source rupture scale and magnitude in western China $[\mathrm{J}]$. Earthquake disaster prevention technology, 10 (2015): 68-76.

3. Zheng Zuoya, Lu Xiushan, Li Kexing. Construction of a class of wavelet basis functions and its application in measurement data processing [J]. Surveying and mapping science, (2007):9-11.

4. Tang Shoufeng, Tong Minming, Pan Yuxiang, He Xinmin, Lai Xiaosong. Analysis of microseismic signal of coal and rock fracture by wavelet signature spectroscopy [J]. Acta instrumentation sinica, (2011): 1521-1527.

5. Li Ying, Zhang Shuzhen, $\mathrm{Xu}$ Kangsheng. Application of wavelet noise reduction method in seismic signal processing $[\mathrm{J}]$. Journal of northwest seismology, (2006): 159-162.

6. S. Mostafa Mousavi, Charles A. Langston. Hybrid Seismic Denoising Using Higher-Order Statistics and Improved Wavelet Block Thresholding [J]. Bulletin of the Seismological Society of America, (2016):1380-1393.

7. Vittoria Bruni, Domenico Vitulano. Wavelet-based signal de-noising via simple singularities approximation. [J]. Signal Processing, 2006. 86(4): 859-876.

\footnotetext{
a Corresponding author: 1009278271@qq.com
} 